\title{
Conocimiento y tratamiento del herpes labial utilizado por adolescentes en Medellín
}

\author{
Teenagers' Knowledge and Treatment of Herpes \\ Labialis in Medellín
}

\author{
Esthefanya Díaz Álvarez, Est., Od. ${ }^{1}$, Shirley Cristina Agudelo Cano, Est., Od. ${ }^{2}$, \\ Eliana María Muñoz Vargas, Est., Od. ${ }^{3}$, Luisa Fernanda Martínez Osorio, Est., \\ Od. ${ }^{4}$, Ángela María Sarrazola Moncada $\bowtie$, MSc. $^{5}$
}

${ }^{1}$ Universidad Cooperativa de Colombia, Medellín y Envigado, Colombia
2 Universidad Cooperativa de Colombia, Medellín y Envigado, Colombia
${ }^{3}$ Universidad Cooperativa de Colombia, Medellín y Envigado, Colombia
${ }^{4}$ Universidad Cooperativa de Colombia, Medellín y Envigado, Colombia
${ }^{5}$ Universidad Cooperativa de Colombia, Medellin y Envigado, Colombia

Facultad de Odontología, Universidad Cooperativa de Colombia, sede Medellín y Envigado, Carrera 42 n. ${ }^{\circ}$ 49-95, Bloque 8, Medellín, Antioquia, Colombia. Correo electrónico: angela.sarrazola@campusucc.edu.co

Recibido: 12 de enero del 2017 Aprobado: 23 de febrero del 2017

Cómo citar este artículo: Díaz-Álvarez E, Agudelo-Cano SC, Muñoz-Vargas EM, Martínez- Osorio LF, Sarrazola-Moncada AM. Conocimiento y tratamiento del herpes labial utilizado por adolescentes en Medellín. Rev Nac Odontol. 2018;13(26):1-7. doi: http://dx.doi.org/10.16925/od.v13i26.2040

\section{Resumen}

Introducción: el profesional del área de la salud debe interesarse por identificar los saberes en torno al proceso salud-enfermedad de las comunidades, con el propósito de promover la salud y prevenir el desarrollo de enfermedades.

Objetivo: describir los conocimientos y tratamientos utilizados por estudiantes adolescentes de una institución educativa pública en Medellín acerca del herpes labial durante el 2012.

Materiales y métodos: estudio observacional, descriptivo. Participaron 133 estudiantes de una institución educativa pública en Medellín mediante un muestreo probabilístico y con selección aleatoria, en el cual se aplicó una prueba de conocimientos y tratamientos sobre herpes labial. Se hizo un análisis estadístico descriptivo univariado. Estudio de riesgo mínimo según Resolución 8430 de 1993 de la Republica de Colombia.

Resultados: la edad promedio fue de 16 años, con una desviación estándar de 1,14, con un mínimo de 14 y un máximo de 23 años. El 50,4\% fueron mujeres, solo el 6\% conoce del herpes labial por fuentes médicas, el 38,3\% desconoce el agente causal, el 65,4\% conoce los medios de transmisión y el 76,7\% lo reconoce visualmente. El 45,9\% tenía dudas sobre los signos y síntomas, el 60,2\% no conoce ningún tratamiento farmacológico, pero refiere conocer el uso de tratamientos no farmacológicos como vaselina, manteca de cacao, químicos, cigarrillo, limón, tomate y sal.

Conclusiones: se detectaron falencias en el conocimiento del herpes labial, su etiología y tratamiento. La fuente principal de conocimiento fue la familia.

Palabras clave: adolescentes, conocimiento, estudiantes, herpes labial, tratamiento. 


\title{
Teenagers' Knowledge and Treatment of Herpes Labialis in Medellín
}

\begin{abstract}
Introduction: A health professional should be interested in identifying communities' knowledge about the health-disease process to promote health and prevent the development of diseases.

Aim: To describe the knowledge and treatments of herpes labialis by adolescent students from a public educational institution in Medellín in 2012.

Materials and methods: Observational, descriptive study. A total of 133 students from a public educational institution in Medellín participated through probabilistic sampling and random selection, and a test of knowledge and treatments of herpes labialis was administered. A univariate descriptive statistical analysis was made. Minimum risk study according to Resolution 8430 of 1993 of the Republic of Colombia.

Results: Ages ranged from 14 to 23 years old with an average age of 16 and a standard deviation of 1.14 $50.4 \%$ were women, only $6 \%$ know about herpes labialis from medical sources, $38.3 \%$ do not know the causative agent, $65.4 \%$ know the means of transmission, and $76.7 \%$ recognize them visually. $45.9 \%$ had doubts about the signs and symptoms, $60.2 \%$ did not know any pharmacological treatment but reported knowing the use of non-pharmacological treatments such as Vaseline, cocoa butter, chemicals, cigarette, lemon, tomato and salt.
\end{abstract}

Conclusions: Deficiencies were detected in the knowledge of herpes labialis, its etiology and treatment. The main source of knowledge was the family.

Keywords: teenagers, knowledge, students, herpes labialis, treatment.

\section{Conhecimento e tratamento do herpes labial utilizados por adolescentes em Medellín}

\section{Resumo}

Introdução: o profissional da área da saúde deve interessar-se por identificar os saberes sobre o processo saúde-doença das comunidades, com o objetivo de promover a saúde e prevenir o desenvolvimento de doenças.

Objetivo: descrever os conhecimentos e tratamentos utilizados por estudantes adolescentes de uma instituição educativa pública em Medellín acerca do herpes labial durante 2012.

Materiais e métodos: estudo observacional e descritivo, do qual participaram 133 estudantes de uma instituição educativa pública de Medellín, por meio de amostragem probabilística e com seleção aleatória, na qual foi aplicado um teste de conhecimentos e tratamentos sobre herpes labial. Fez-se uma análise estatística descritiva univariada. Estudo de risco mínimo, segundo a Resolução 8.430 de 1993 da República da Colômbia.

Resultados: a idade média foi de 16 anos, com um desvio-padrão de 1,14, com um mínimo de 14 e um máximo de 23 anos. 50,4 \% foram mulheres; somente $6 \%$ conhecem o herpes labial por fontes médicas, 38,3\% desconhecem o agente causal; 65,4 \% conhecem os meios de transmissão, e 76,7 \% o reconhecem visualmente. 45,9 \% expressaram dúvidas sobre os sinais e sintomas; 60,2 \% não conhecem nenhum tratamento farmacológico, mas referem conhecer uso de tratamentos não farmacológicos como vaselina, manteiga de cacau, químicos, cigarro, limão, tomate e sal.

Conclusões: foram detectadas falhas no conhecimento do herpes labial, em sua etiologia e tratamento. A fonte principal de conhecimento foi a família.

Palavras-chave: adolescentes, conhecimento, estudantes, herpes labial, tratamento. 


\section{Introducción}

El profesional del área de la salud debe interesarse por identificar los saberes en torno al proceso salud-enfermedad de las comunidades, con el propósito de promover la salud y prevenir el desarrollo de enfermedades [1]. La educación en salud se puede impartir en los grupos poblacionales, direccionada por sus etapas de desarrollo. Los jóvenes en la adolescencia se enfrentan a constantes cambios, experiencias e influencias hormonales que pueden favorecer el desarrollo o la expresión clínica de enfermedades, entre ellas el virus del herpes simple si de manera previa han sido expuestos a este.

El herpes labial es una enfermedad infectocontagiosa que se presenta en $20-40 \%$ de la población general. Es de fácil propagación, y una vez que una persona entra en contacto con el virus, queda latente en el sistema nervioso central, reactivándose cada vez que se presenten episodios de estrés, depresión, fatiga, exposición al sol y cambios hormonales [2].

El virus herpes simple tipo 1 (vHs-1), también conocido como "herpes labial", es el agente etiológico de las lesiones vesiculares de la mucosa oral; este virus presenta una amplia variedad de localizaciones anatómicas, usualmente de la cintura hacia arriba (hígado, pulmones, ojos y el sistema nervioso central), y al incluir los genitales, se asocia también a infecciones cruzadas. Estas infecciones pueden ser graves, especialmente en personas con inmunosupresión [3].

La infección primaria se da por la inoculación del vis-1 en las mucosas o en los sitios de la piel que permiten la entrada del virus en la epidermis, en la dermis y en las terminaciones nerviosas sensoriales y autonómicas. Una vez que se ha producido la infección por el virus del herpes simple (vHs), este vive en un estado latente en los cuerpos de las células nerviosas en las neuronas ganglionares y se puede reactivar. La frecuencia y la gravedad de reactivación están determinadas por muchos factores, incluyendo la inmunodeficiencia o el estrés [4].

El inicio de la enfermedad clínica suele ser repentino, con la aparición de múltiples lesiones vesiculares características superpuestas sobre una base eritematosa e inflamatoria. La infección primaria también puede estar o no estar asociada con síntomas sistémicos, como fiebre y malestar general, y es considerablemente menor su sintomatología, el número y el tamaño de las lesiones en los episodios de la reactivación del virus [5]. Aunque los síntomas pueden ser graves, la mayoría de las infecciones primarias por vHs-1 son asintomáticas [6].

En contraste con la infección primaria vHS1, la reactivación del virus rara vez se asocia con signos o síntomas sistémicos, excepto para linfoadenopatía local. La mayoría de los pacientes son conscientes de los síntomas prodrómicos que anuncian la aparición de un episodio de reactivación, como dolor, ardor, hormigueo y prurito. Estos síntomas pueden aparecer entre seis y 53 horas antes de la aparición de las primeras vesículas [7].

Las lesiones vesiculares alcanzan a ser dolorosas y duran cinco días en los inmunocompetentes, y de diez a catorce días en los inmunocomprometidos. Las lesiones tienden a agruparse en un solo sitio anatómico y pueden reaparecer en el mismo lugar o zonas adyacentes [8]; en las zonas adyacentes, se dan debido a la propagación centrífuga a través de los nervios periféricos, diseminación contigua a nuevas células epiteliales, y ocurren en lugares distantes por medio de una nueva infección primaria, autoinoculación e infección diseminada rara vez [9].

Las recurrencias, también conocidas como reactivaciones del virus, pueden darse por condiciones hormonales, periodos de estrés, ansiedad, exposiciones prolongadas a la luz solar o traumatismos; y ocurren frecuentemente una vez al mes en el 24\% de los casos o en menor frecuencia (aproximadamente dos veces al año) en el 19\%. Al involucrar las zonas más comunes (como la cavidad oral y los labios con 85\%), las lesiones progresan de vesícula a la corteza en unos ocho días con disminución significativa del dolor después de las primeras 24 horas de su aparición [7].

Para el herpes labial recurrente, el tratamiento ideal es aciclovir en crema al 5\%, cinco veces al día, durante cinco días. Su utilización puede abortar las lesiones y acelerar la curación siempre que sea administrado antes de la aparición de vesículas. Una vez establecido el tratamiento con aciclovir sistémico, resulta bastante efectivo en una dosis de $200 \mathrm{mg}$, cinco veces al día mientras existan lesiones, incluso en pacientes inmunosuprimidos. Es muy importante la hidratación oral y pueden emplearse anestésicos tópicos en forma de colutorios y/o analgésicos sistémicos. Se recomienda mantener una buena higiene oral para evitar posibles sobreinfecciones [10]. 
Para tratar sus manifestaciones clínicas, los jóvenes también están dados a automedicarse, pero en algunos casos, dado el desconocimiento que se tiene sobre el herpes labial, se pueden producir sobreinfecciones y alergias, lo cual prolonga la recuperación y afecta la apariencia física [11].

La adolescencia es una etapa en la que fácilmente puede haber contagio y propagación del virus del herpes. Es importante indagar sobre el conocimiento y tratamiento para el herpes labial utilizado por los jóvenes, a fin de proporcionar pautas para la educación en salud y prevención del contagio y difusión de la enfermedad.

El propósito del presente estudio fue describir los conocimientos y tratamientos utilizados por estudiantes adolescentes de una institución educativa pública en Medellín, acerca del herpes labial en el 2012.

\section{Materiales y métodos}

Se realizó un estudio observacional, descriptivo. La población fue de 240 estudiantes adolescentes de noveno y décimo de la Institución Educativa Pública Yermo y Parres en Medellín. La selección de la muestra se hizo mediante un muestreo aleatorio simple, con un error del 5\%, un nivel de confianza del $95 \%$ y una proporción del 50\% para un total de 147 estudiantes.

Los criterios de inclusión fueron: estudiantes activos, matriculados en los grados noveno y décimo, y los criterios de exclusión fueron: estudiantes que no decidieron participar en el estudio o estaban ausentes al momento de recolectar la información.

Para obtener la información, se aplicó una encuesta de doce preguntas con escala tipo Likert sobre los conocimientos del herpes labial y los tratamientos utilizados por los adolescentes, previa prueba piloto que permitió ajustar el instrumento de medición.

Para el procesamiento y el análisis de la información, se creó una base de datos, se verificó la validez de la información registrada en los formularios con la información diligenciada en la base de datos, se realizó un análisis univariado mediante el programa estadístico SPSs 21.0 y se presentaron los resultados en frecuencias y porcentajes, mediante gráficos y tablas.
El desarrollo de este proyecto contó con la aprobación de la institución educativa pública a través de su inclusión en actividades académicas en el área de ciencias naturales. Los participantes diligenciaron consentimiento informado. El estudio es considerado de riesgo mínimo según la Resolución 008430 de 1993 (Ministerio de Salud de Colombia).

\section{Resultados}

Se evaluaron 133 adolescentes de noveno y décimo, en noviembre del 2012. La edad promedio fue de 16 años, con una desviación estándar de 1,14, con un mínimo de 14 y un máximo de 23 años. No se logró la participación de catorce estudiantes en la investigación, pues no asistieron el día de la recolección de la información.

El 50,4\% (67) fueron mujeres, la fuente de conocimiento fue la familia en el 53,4\% de la muestra, lo cual es de resaltar pues es la familia la primera línea de comunicación con los adolescentes. En segundo lugar, no responde el 26,3\%, seguido de obtención de la información por amigos (8,3\%), por personal médico (6\%), por medios de comunicación (periódicos, libros, revistas y televisión) $(3,8 \%)$ y por sus profesores $(2,3 \%)$.

En cuanto a los conocimientos del herpes labial, el 50,4\% está de acuerdo en que el herpes se puede transmitir, el 40,6\% está de acuerdo con la sintomatología que presenta ante su manifestación clínica (picazón, ardor y resequedad), aunque dicen desconocer con precisión que su etiología es viral.

Respecto al reconocimiento visual del herpes, se encontró que el 76,7\% identificó correctamente la imagen del popularmente llamado "fuego" (véase la Figura 1).

El 62\% de los estudiantes reportó no utilizar tratamientos farmacológicos locales, pero al preguntar sobre sustancias aplicadas para controlar las manifestaciones clínicas del herpes labial, expresaron usar hielo, sustancias aislantes (vaselina), sustancias humectantes (crema de limón, Chapstick y manteca de cacao), sustancias químicas (removedor de esmalte de uñas, hipoclorito de sodio, ceniza de cigarrillo, alcohol con talco, piedra lumbre), sustancias estabilizadoras de pH (bicarbonato de sodio y sal), y sustancias naturales (tomate con azúcar, limón). 


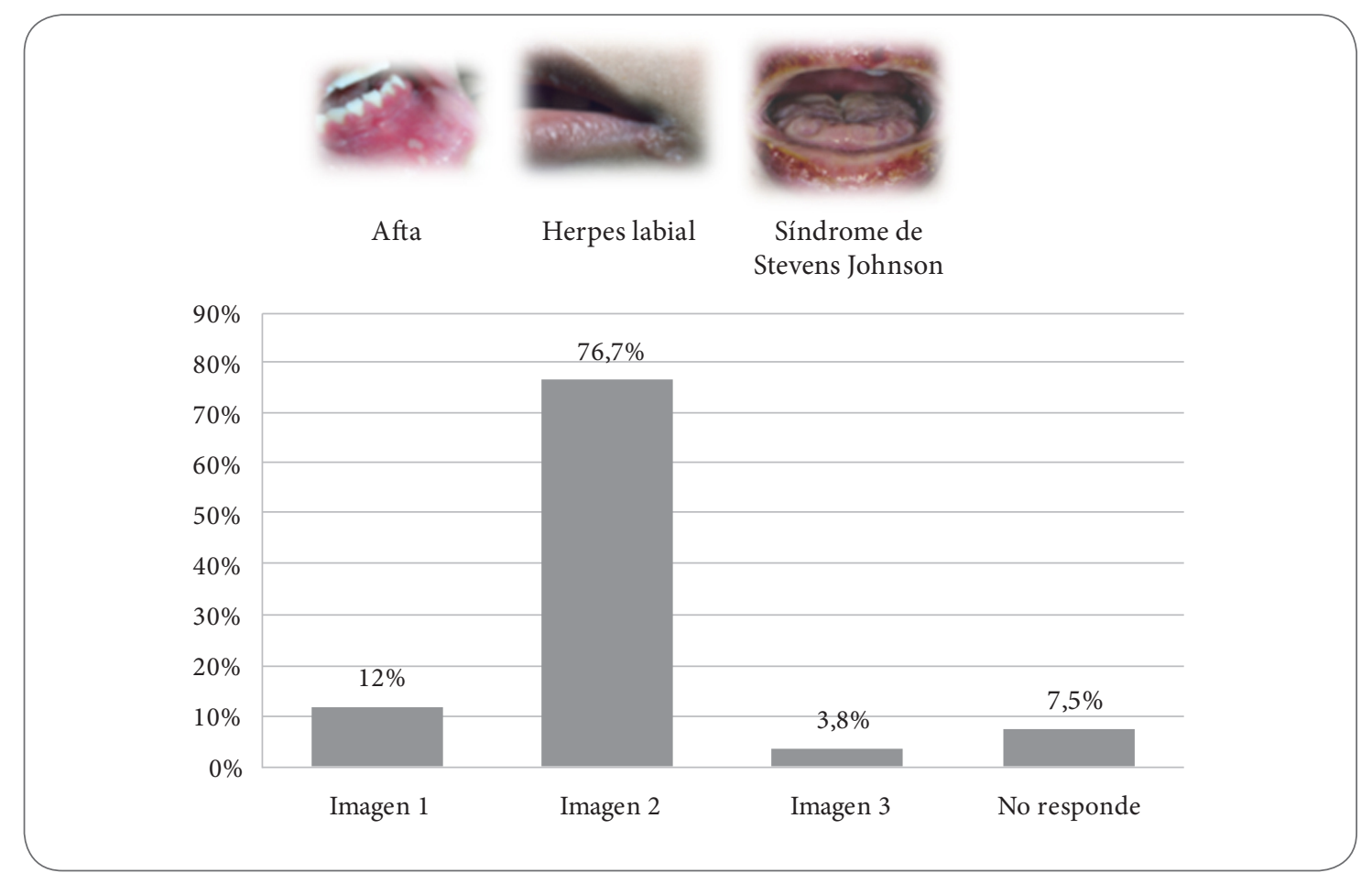

Figura 1. Reconocimiento visual del herpes labial de los adolescentes participantes en el estudio Fuente: elaboración propia

\section{Discusión}

Tener un acercamiento a las concepciones teóricas de las comunidades sobre el proceso de salud y enfermedad sigue siendo una actividad importante y básica, a fin de comprender su actuar y reorientar lo que sea requerido para mejorar la condición de salud, sobre todo en la población de bajos recursos socioeconómicos.

En Colombia, se encuentra reportado un estudio realizado en Bogotá en adultos jóvenes en el que la prevalencia de las lesiones herpéticas labiales fue del $38,7 \%$, con desarrollo de la lesión en su mayoría una vez al año, sin diferencias entre sexos y con mayor prevalencia de lesiones labiales en los estratos 1 y 2; por lo cual resalta la importancia de establecer controles para evitar su diseminación y prevenir daños más severos en población de riesgo [12].

Los resultados de este estudio muestran que los adolescentes tienen conocimientos sobre el herpes labial, sus síntomas y reconocen (en su mayoría) la manifestación clínica. Esto es similar a la investigación en estudiantes de bachillerato del Colegio
Viet Nam de La Habana, Cuba [13], en la que reportan un conocimiento del herpes simple del $63,3 \%$, y que difiere de un estudio realizado en Europa, según el cual los jóvenes tenían más conocimiento del vir/sida y del virus del papiloma humano que del herpes genital y menos aún del labial [14].

En un estudio realizado en Venezuela, también se evidencia que los jóvenes tienen nociones de conocimiento sobre enfermedades de transmisión sexual, siendo sus familias la fuente de información principal en el 46,2\% [15]. Similar al resultado del presente estudio, en el que la obtención de información acerca del herpes labial de los adolescentes fue en su mayoría de la familia, en el $54 \%$.

Este resultado también fue similar al reportado por un estudio realizado en Italia [2], en el cual constataron que la mayoría de las personas obtenían el conocimiento sobre el herpes labial de los padres/familiares y amigos/conocidos, y solo una minoría obtenía información del personal de la salud [2]. Esto también coincide con el estudio realizado en los estudiantes de bachillerato del Colegio Viet Nam de La Habana, Cuba, en el que la familia ocupa el mayor porcentaje, con el 53,4\%; seguida de 
los amigos, con 8,3\%; los profesores, con 2,3\%; los medios de comunicación (libros, periódicos y televisión) con 3,8\%; y la fuente médica, con $6 \%$ [11].

La incertidumbre en cuanto a la etiología del herpes y su tratamiento cuando no acceden a orientación médica lleva a los adolescentes a realizar prácticas inadecuadas que atentan contra la integridad física, similar a lo encontrado en un estudio realizado en Estados Unidos. Esto debe ser de interés para los profesionales del área de la salud, lo cual corrobora la importancia de conocer las prácticas en salud para orientar este conocimiento con la educación para la salud y la prevención del contagio y la generación de sobreinfecciones por mal manejo [16].

El $60,2 \%$ de los estudiantes con edad promedio de 16 años, una edad mínima de 14 años y máxima de 23 años, reportaron no utilizar tratamientos farmacológicos locales, a diferencia de un estudio realizado en Bogotá, en el que el 52,7\% refirió utilizar alguna sustancia para su tratamiento, siendo los antivirales tópicos los de mayor uso, pero en jóvenes de 18 a 30 años, con promedio de 22,9 años. Esta información sigue evidenciando la necesidad de promover actividades educativas a cargo de profesionales del área de la salud [12].

\section{Conclusiones}

Los adolescentes participantes en el estudio tienen cierto conocimiento sobre los síntomas, la transmisión y la identificación visual del herpes labial, pero se detectaron falencias en el conocimiento de la etiología y prácticas inadecuadas de tratamiento.

La fuente principal de conocimiento sobre el herpes labial fue la familia, lo cual motiva a dirigir programas educativos a los padres sobre diferentes temáticas en salud, entre ellas la promoción de la salud y la prevención de transmisión de enfermedades.

Esta investigación se incluyó en el proyecto ambiental de una institución educativa pública en Medellín, como actividad para promover la salud y prevenir el contagio de la enfermedad, con lo que se propicia la generación de una actitud responsable frente al herpes labial por parte de los estudiantes desde la institución educativa.

\section{Referencias}

[1] Masoe AV, Blinkhorn AS, Taylor J, Blinkhorn FA. Factors that influence the preventive care offered to adolescents accessing Public Oral Health Services, Nsw, Australia. Adolesc Health Med Ther. 2015;6:10113. doi: http://dx.doi.org/10.2147/AHMT.S84332.

[2] Pica F, Volpi A. Public awareness and knowledge of herpes labialis. Journal of Medical Virology. 2012;84(1):132-7. doi: http://dx.doi.org/10.1002/jm v. 22233 .

[3] Fatahzadeh M, Schwartz RA. Human herpes simplex labialis. Clinical and Experimental Dermatology. 2007;32(6):625-30. doi: http://dx.doi.org/10.1111/j. 1365-2230.2007.02473.x.

[4] Freeman ML, Sheridan BS, Bonneau RH, Hendricks RL. Psychological stress compromises CD8+ T cell control of latent herpes simplex virus type 1 infections. J Immunol. 2007;179(1):322-8. doi: https:// doi.org/10.4049/jimmunol.179.1.322.

[5] Oliver L, Wald A, Kim M, Zeh J, Selke S, Ashley R, et al. Seroprevalence of herpes simplex virus infections in a family medicine clinic. Arch Fam Med. 1995;4(3):228-32.

[6] Johnson RE, Nahmias AJ, Magder LS, Lee FK, Brooks CA, Snowden CB. A seroepidemiologic survey of the prevalence of herpes simplex virus type 2 infection in the United States. N Engl J Med. 1989; 321(1):7-12. doi: http://dx.doi.org/10.1056/NEJM19 8907063210102 .

[7] Spruance SL, Overall JC, Kern ER, Krueger GG, Pliam V, Miller W. The natural history of recurrent herpes simplex labialis: Implications for antiviral therapy. N Engl J Med. 1977;297(2):69-75. doi: http://dx.doi.org/10.1056/NEJM197707142970201.

[8] Glogau R, Hanna L, Jawetz E. Herpetic whitlow as part of genital virus infection. J Infect Dis. 1977; 136(5):689-92. doi: https://doi.org/10.1093/infdis/ 136.5.689.

[9] Steinar I, Kennedy PG. Herpes simplex virus latent infection in the nervous system. J Neurovirol. 1995;1(1):19-29.

[10] Pousa Castro X, Bascones Martínez A. Herpesvirus. Av Odontoestomatol. 2011;27(1):11-24.

[11] St Pierre SA, Bartlett BL, Schlosser BJ. Practical management measures for patients with recurrent herpes labialis. Skin Therapy Lett. 2009;14(8):1-3.

[12] Barrientos S, Velosa J, Rodríguez A. Prevalencia de herpes labial recurrente en población de 18 a 30 años de edad en Bogotá, Colombia. Univ Odontol. 2014;33(71). doi: http://dx.doi.org/10.11144/Javeriana.uo33-71.phlr. 
[13] Puentes Rizo E, Enríquez Domínguez B, Rodríguez de Celis Y, Correa Jáuregui M. La sexualidad en adolescentes de la secundaria básica "Viet Nam". Rev Cubana Med Gen Integr [Internet]. 2012 [citado 2015 jul 8]; 28(4):599-610. Disponible en: http:// scielo.sld.cu/scielo.php?script=sci_arttext\&pi$\mathrm{d}=$ S0864-21252012000400004\&lng=es.

[14] Samkange-Zeeb FN, Spallek L, Zeeb H. Awareness and knowledge of sexually transmitted diseases (sTDs) among school-going adolescents in Europe: A systematic review of published literature. BMC Public Health. 2011;11:727. doi: http://dx.doi.org/ 10.1186/1471-2458-11-727.
[15] Corona Lisboa J, Ortega Alcalá J. Comportamiento sexual y conocimiento sobre infecciones de transmisión sexual en estudiantes venezolanos de un liceo del municipio de Miranda. Medisan [Internet]. 2013 [citado 2015 jul 9]; 17(1):78-85. Disponible en: http://scielo.sld.cu/scielo.php?script=sci_arttext\&pid=S1029-30192013000100011\&lng=en.

[16] Beydoun HA, Dail J, Ugwu B, Boueiz A, Beydoun MA. Socio-demographic and behavioral correlates of herpes simplex virus type 1 and 2 infections and co-infections among adults in the USA. In J Infect Dis. 2010;14(Suppl 3):e154-60. doi: http://dx.doi. org/10.1016/j.ijid.2009.12.007. 